\title{
Personalising docetaxel and G-CSF schedules in cancer patients by a clinically validated computational model
}

\author{
O Vainas', S Ariad'2, O Amir', W Mermershtain², V Vainstein ${ }^{1,3}$, M Kleiman', O Inbar', R Ben-Av', \\ A Mukherjee ${ }^{4}$, S Chan $^{4}$ and Z Agur*, ${ }^{*, 3}$
}

'Optimata Ltd, 7 Abba Hillel Street, Ramat-Gan 52522, Israel; '2Department of Oncology, Soroka University Medical Center, Be'er Sheba 84 I 01 , Israel; ${ }^{3}$ Institute for Medical BioMathematics, 10 Hate'ena Street, P.O. Box 282, Bene Ataroth 6099I, Israel; ${ }^{4}$ Department of Clinical Oncology and Histopathology, Nottingham City Hospital, Hucknall Road, Nottingham NG5 IPB, UK

BACKGROUND: This study was aimed to develop a new method for personalising chemotherapeutic and granulocyte colony-stimulating factor (G-CSF) combined schedules, and use it for suggesting efficacious chemotherapy with reduced neutropenia.

METHODS: Clinical data from 38 docetaxel (Doc)-treated metastatic breast cancer patients were employed for validating a new pharmacokinetic/pharmacodynamics model for Doc, combined with a mathematical model for granulopoiesis. An optimisation procedure was constructed and used for selecting improved treatment schedules.

RESULTS: The combined model accurately predicted observed nadir timing $(r=0.99)$, grade 3 or 4 neutropenia (86\% success) and neutrophil counts over time in individual patients $(r=0.63)$, and showed robustness to CYP3A-induced variability in Doc clearance. For average patients, the predicted optimal support for the standard chemotherapy regimen, Doc $100 \mu \mathrm{g} \mathrm{m}^{-2}$ tri-weekly, is G-CSF, $300 \mu \mathrm{g}, \mathrm{Q} I \mathrm{D} \times 3$, starting day 7 post-Doc. This regimen largely moderates chemotherapy-induced neutrophil nadir and neutropenia duration. The more intensive Doc dose, $150 \mathrm{mg} \mathrm{m}^{-2}$, is optimally supported by the slightly less cost-effective G-CSF $300 \mu \mathrm{g}$, QID $\times 4,5$ days post-Doc. The latter regimen is optimal for borderline patients (2000 neutrophils per $\mu$ l) under Doc, $100-150 \mathrm{mg} \mathrm{m}^{-2}$ tri-weekly.

CONCLUSIONS: The new computational method can serve for tailoring efficacious cytotoxic and supportive treatments, minimising side effects to individual patients. Prospective clinical validation is warranted.

British Journal of Cancer (2012) 1 07, 814-822. doi:I0.1038/bjc.2012.316 www.bjcancer.com

Published online 19 July 2012

(c) 2012 Cancer Research UK

Keywords: granulocyte colony-stimulating factor; neutropenia; Doc; mechanistic PK/PD; mathematical modelling; optimisation

The major dose-limiting toxicity for docetaxel (Doc), neutropenia (Cortes and Pazdur, 1995), is associated with considerable morbidity, mortality and excessive hospital admissions (Crawford et al, 2004). Docetaxel monotherapy $\left(60-100 \mathrm{mg} \mathrm{m}^{-2}\right.$, i.v., Q21D), a preferred first-line therapy for recurrent and metastatic breast cancer (MBC), often results in grade 3 or 4 neutropenia (Montero et al, 2005). The appearance of grade 3 or 4 neutropenia frequently leads to delayed chemotherapy administration or dose reduction, both having been associated with worse clinical outcomes (Cameron, 2009; Krell and Jones, 2009).

Increased overall dose intensity of Doc, postulated to increase efficacy (Bruno et al, 1998; Bruno et al, 2003), can compromise safety of the patient. In patients experiencing grade 3 or 4 Docrelated toxicity, granulocyte colony-stimulating factor (G-CSF) can be used for alleviating neutropenia. When the standard G-CSF regimen is applied (300 $\mu \mathrm{g}$ administration starting day 1 post Doc for 5-6 consecutive days) in conjunction with $100 \mathrm{mg} \mathrm{m}^{-2}$ biweekly Doc, no grade 4 neutropenia is reported in locally advanced breast cancer patients (Paciucci et al, 2002), or in advanced NSCLC patients (Gridelli et al, 2000). Nevertheless, an improved schedule for the combined Doc and G-CSF therapy is yet to be determined.

*Correspondence: Dr Z Agur; E-mail: agur@imbm.org

Received 19 March 2012; revised 18 June 2012; accepted 26 June 2012; published online 19 July 2012
Such problems are usually addressed by costly and lengthy clinical trials, whose conclusions may be compromised by the number of recruited patients and the number of tested regimens. In contrast, using mathematical models may be a fast and inexpensive way to predict beneficial Doc and G-CSF combination therapy out of a large space of clinically relevant regimens.

Conventional pharmacokinetic/pharmacodynamics (PK/PD) models, although mandatory for predicting instantaneous drug activity, cannot serve this end. Such models do not predict the long-term effects of drugs, which depend on the dynamics of the affected biological process, in the present case - granulopoiesis. In contrast, physiological mathematical models of granulopoiesis can be used for studying the effects of chemotherapy (Friberg et al, 2002; Shochat et al, 2002; Engel et al, 2004; Vainstein et al, 2006), radiation therapy (Schmitz et al, 1990; Schmitz et al, 1993), pathological hematopoiesis (Mackey et al, 2003; Dingli et al, 2009), bone marrow (BM) transplantation (Ostby et al, 2003) or postchemotherapy G-CSF support (Vainstein et al, 2005, 2006; Shochat and Rom-Kedar, 2008; Foley and Mackey, 2009), and the allometric scalability across mammals in normal and malignant hematopoiesis (Pacheco et al, 2009).

In this work, we developed a computational method for optimising chemotherapy and G-CSF regimens in individual patients. The method employs our previously constructed mathematical model for granulopoiesis (Vainstein et al, 2005), which differs from other granulopoiesis models in accounting for the complex dynamics of 
mitotic and non-mitotic BM progenitors, with explicit description of cell cycle phase transition in the mitotic compartments. This allows for a faithful representation of the action of cell cycle phase-specific drugs, such as Doc. The model, which includes the secretion, diffusion, clearance and specific interactions of G-CSF with cells in the different granulopoiesis compartments, is new in considering both short- and long-term effects of G-CSF on the BM, both in neutropenic and in healthy subjects.

To validate the granulopoiesis model and use it to suggest safer chemotherapy regimens, a mechanistic PK/PD model of Doc was combined with the granulopoiesis model, for which the parameters were literature-based (Vainstein et al, 2005). The integrated granulopoiesis-PK/PD model was adjusted to describe a population of MBC patients, treated by Doc monotherapy (denoted population-specific model). The population-specific model was then validated on an independent set of Doc patients from the same population. Following validation, the model was integrated with a new optimisation procedure, thus enabling the identification of improved Doc/G-CSF regimens. Specifically, two optimisation processes were performed on a range of potential regimens, one in which the G-CSF regimen was optimised for the standard tri-weekly Doc regimen, and a second in which the G-CSF regimen was optimised for a potential high-Doc regimen $\left(150 \mathrm{mg} \mathrm{m}^{-2}\right.$ triweekly). This process was performed for patients with various initial neutrophil counts.

\section{PATIENTS AND METHODS}

\section{Patients}

Weekly blood counts were collected from 38 Caucasian MBC patients from two sites, treated by tri-weekly or weekly Doc regimens; neutropenic patients were sampled bi-weekly until recovery (Nottingham City Hospital, Nottingham, UK; Soroka Medical Centre, Be'er Sheva, Israel; see details in Table 1). Using the Nyquist-Shannon sampling theorem, the sampling was found sufficient for our modelling purposes (Jerri, 1979; Shannon, 1949). Patients from both sites were mixed and randomly divided into a set of data to be used for calibrating model parameters $(N=12$; denoted training set) and a set of data to be used for model validation $(N=26$; denoted validation set). Docetaxel schedules and neutrophil baselines (median 5080 neutrophils per $\mu \mathrm{l}$; range 1800-15500) were used as model input. Individual plasma Doc measurements were not collected, and prior chemotherapy and performance status, only partially recorded, were not included.

\section{General granulopoiesis model}

The mathematical model employed describes neutrophil development in the BM, from myeloid progenitors (including colony-

Table I Patients demographics for MBC study population

\begin{tabular}{|c|c|c|}
\hline & $\begin{array}{l}\text { Nottingham } \\
\text { City Hospital, } \\
\text { UK }(N=\text { I } 2)\end{array}$ & $\begin{array}{c}\text { Soroka University } \\
\text { Hospital, Israel } \\
(N=26)\end{array}$ \\
\hline Age & $51(36-76)$ & $55(30-80)$ \\
\hline Female & 12 & 25 \\
\hline Male & - & I \\
\hline Weight & $64(45-80)$ & $68(45-98)$ \\
\hline Height & $162(150-170)$ & $160(\mid 48-175)$ \\
\hline Ethnic group & Caucasians & Caucasians \\
\hline Docetaxel treatment duration (days) & $152(\mid 14-274)$ & $106(38-354)$ \\
\hline Treatment regimen & Q21D & Q21D or Q7Da \\
\hline Dose $\left(\mathrm{mg} \mathrm{m}^{-2}\right)$ & $100(67.5-100)$ & $36(23-101)$ \\
\hline
\end{tabular}

Abbreviations: $\mathrm{MBC}=$ metastatic breast cancer; $\mathrm{Q} 2 \mathrm{ID}=$ docetaxel administered every 3 weeks; Q7D = docetaxel administered every 7 days. ${ }^{a}$ Annals of Oncologyannonc.oxfordjournals.org forming unit (CFU) of granulocyte, erythrocyte, monocyte and megakaryocyte; CFU of granulocyte and monocyte; and CFU of granulocyte inflow, through proliferating neutrophil progenitors (myeloblasts, promyelocytes and myelocytes) and post-mitotic progenitors to blood neutrophils (Figure 1). The mathematical model features an explicit cell cycle in the mitotic compartments and receptor-mediated G-CSF clearance. The G-CSF is taken as a feedback molecule governing BM maintenance of a steady neutrophil level in the blood. The model includes G-CSF secretion, diffusion, clearance and interactions with different cell compartments in the normal neutrophil development. The parameters of the general granulopoiesis model were estimated based on an extensive set of experimental data, including radioactive labelling of BM cell compartments in vivo, and blood neutrophil profiles in normal population-specific conditions, as well as in cancer patients subject to chemotherapy. The parameters of G-CSF PK/ PD were estimated based on experimental data from neutropenic patients and healthy volunteers administered with G-CSF (Vainstein et al, 2005).

\section{Doc PK model}

For calculating the concentration-time profiles, we assumed a three-compartment population mean Doc PK model with linear elimination (Bruno et al, 1996). The parameters of the Doc PK model were estimated using data taken from a study with a dose of $100 \mathrm{mg} \mathrm{m}^{-2}$ Doc administered in a $1 \mathrm{~h}$ i.v. infusion (van Zuylen et al, 2000) and then validated on independent data (Baker et al, 2004) (see also Supplementary Material and Figure S1 therein).

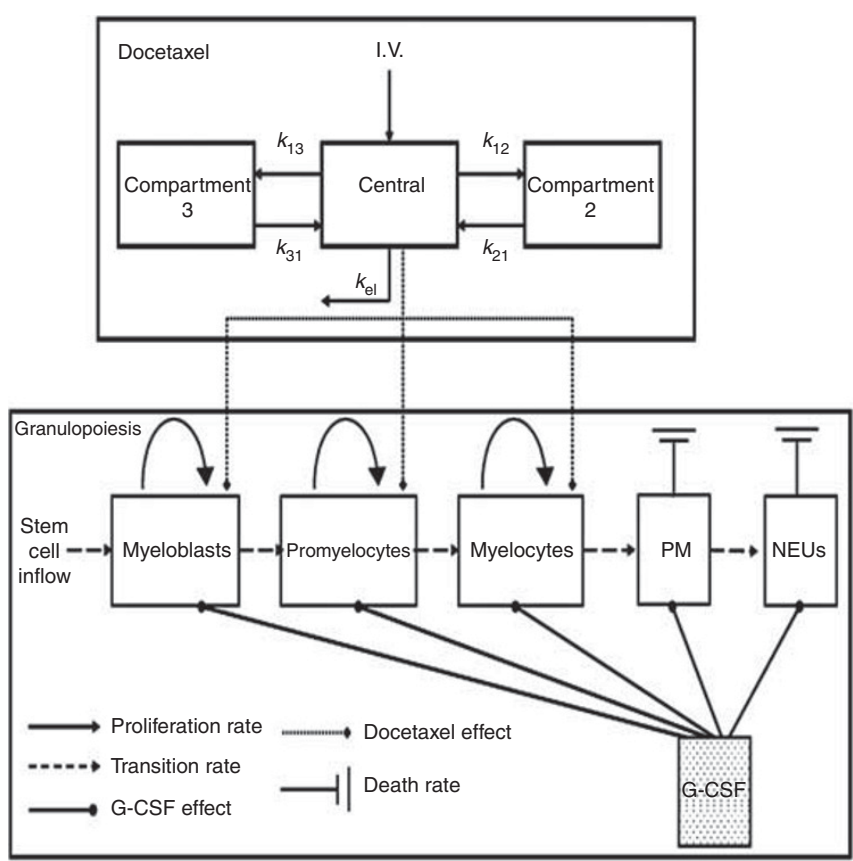

Figure I Schematic description of the combined docetaxel/granulopoiesis model. Docetaxel (upper box) is represented by a three-compartment PK model, arrows represent exchange constants of the drug between the central and peripheral compartments $\left(k_{12}, k_{21}, k_{13}, k_{31}\right)$ and the elimination rate from the body $\left(k_{e}\right)$. Granulopoiesis (lower box) is described as a pipeline initiated by myeloid progenitors (including colony forming unit of granulocyte, erythrocyte, monocyte and megakaryocyte; CFU of granulocyte and monocyte; and CFU of granulocyte) inflowing to the myeloblasts compartment, then, sequentially, differentiating into promyelocytes, myelocytes, post-mitotic (PM) BM cells, and finally being released to blood as mature neutrophils (NEUs). G-CSF accelerates proliferation, transition through the mitotic compartment, release of post-mitotic cells to blood and their apoptosis. Docetaxel targets cells in the mitotic compartments. 


\section{Doc PD model}

The effects of Doc on granulopoiesis (Figure 1) were modelled as a direct killing of neutrophil-proliferating progenitors, myeloblasts, promyelocytes and myelocytes - the most likely targets of Doc in granulopoiesis (Ozawa et al, 2007). The effect of Doc on more primitive hematopoietic compartments was ignored in our model, because the majority of primitive hematopoietic cells are in the G0 phase (Alenzi et al, 2009), and their proliferation due to chemotherapyinduced BM depletion (Hardy and Balducci, 1985) occurs 2-3 days after chemotherapy administration, when Doc concentration is already low. For this reason, cell-cycle-specific drug, such as taxanes, are expected to exert little effect on most primitive cells.

Each Doc effect was related to Doc plasma concentration over time as described in equation (Equation 1):

$$
E(C)=\frac{E_{\max } C^{m}}{C^{m}+C_{\text {nor }}^{m}},
$$

where $E$ is the measured effect at the given concentration $C$ (in units of the fraction of killed cells out of total cell number in each compartment). The maximal possible effect is $E_{\max }$, where $E_{\max }$ is determined by curve fitting to the experimental data, with values ranging from 0 to 1 . The drug concentration, which produces an effect equalling half of $E_{\max }$ is $C_{\text {nor }}$; the curve slope at the point $\left[C_{\text {nor }} ; E\left(C_{\text {nor }}\right)\right]$ is $\left(m E_{\max }\right) /\left(4 C_{\text {nor }}\right)$.

\section{Population-specific model}

The population-specific model was created by first incorporating the PK/PD model of Doc into the general granulopoiesis model. Population-specific model parameters were estimated via least squares curve fitting to clinical data from the training set, using a cross-entropy search algorithm (Kroese et al, 2006). This process resulted in a single parameter set, common for all patients, excluding their initial baseline neutrophil counts and their treatment regimen.

\section{Model validation}

Model validation was performed by comparing model predictions to clinical results of individual patients from the validation set (Esbensen, 2002; Bonate, 2006). The following criteria were used to assess the accuracy in the validation set: (i) accuracy in predicting nadir day (nadir day defined as the lowest observable neutrophil count at each cycle), (ii) accuracy in predicting the occurrence of grade 3 or 4 neutropenia and (iii) accuracy in predicting neutrophil counts over time (denoted neutrophil profile).

\section{Influence of inter-patient PK variability on prediction accuracy}

We examined the influence of CYP3A inter-individual variability (Hirth et al, 2000) on the accuracy of the model by varying the PK parameter mostly affected by this variability, namely Doc clearance. The model was simulated on a population with individual clearance values, randomly sampled from a log normal distribution within the experimentally observed range of $5.4-29.11^{-1} \mathrm{hr}^{-1} \mathrm{~m}^{2}$ (Hirth et al, 2000 ), and the resulting nadir timing and grade 3 or 4 neutropenia were compared with that of the population-specific model for a patient with population-average PK parameters (Supplementary Table S2).

\section{Treatment optimisation}

To identify the Doc and G-CSF combination schedule, which maximises efficacy and minimises neutropenia, our developed treatment optimisation method (Agur et al, 2006) was used. This method incorporates four main elements: (i) a population-specific mathematical model, (ii) a regimen space over which the model is simulated, (iii) a scoring function (SF; denoted also as the fitness function), which grades the expected value of each regimen over the defined space and (iv) a search algorithm. In this work, the regimen space had G-CSF doses ranging between 0 and $480 \mu \mathrm{g}$ per day, with dosing intervals of 1 or 2 days, for $0-10$ consecutive daily applications during each Doc cycle. The optimisation process, as described above, was performed for the general granulopoiesis model with normal and borderline grade 1 initial neutrophil counts of 4250 and 2000 neutrophils per $\mu \mathrm{l}$, respectively, using the cross entropy search algorithm (Kroese et al, 2006).

Scoring function The SF is a function of the G-CSF regimen designed to minimise neutropenia concurrently with the minimisation of G-CSF administration (dose and number of applications). Because of the different toxicity vs G-CSF cost/adminstration tradeoffs, the $\mathrm{SF}$ is formulated as a weighted average of toxicity SF $\left(S F_{\mathrm{TX}}\right)$ and cost SFs $\left(S F_{\text {Cost }}\right)$. These $S F_{\mathrm{TX}}$ and $S F_{\mathrm{Cost}}$ are bound between 0 and 1 , such that the final SF satisfies $S F \in[0,1]$. The toxicity weights and cost weights were set to be 1 and 0.05 , respectively, to ensure that the improved regimens will have low toxicity and will have a minimal amount of G-CSF administrations.

The $S F_{\mathrm{TX}}$ is in turn also a weighted average of five functions $\in[0,1]$, each representing different clinical parameters, such as the length of each patient's longest grade 3 or 4 event, the average length of each patient's grade 3 or 4 event and the percent of the population with grade 2 or higher toxicity at the onset of any cycle. Definitions of neutropenia grades were taken from the NCI Common Terminology Criteria for Adverse Events and Common Toxicity Criteria v4.0 (http://ctep.cancer.gov/protocol Development/electronic_applications/ctc.htm).

The $S F_{\text {Cost }}$ is a weighted average of the cost of treatment in terms of the number of days a patient needs to come to the hospital to be administered treatment, normalised is the number of days in a treatment cycle and the cumulative dose multiplied by the drug cost per unit normalised by the maximum possible cost. As the nuisance of coming into the clinic for drug administration is considered more substantial than the cost of G-CSF, the administration and unit cost elements of the cost function are given weight of 2 and 1 , respectively. The $S F_{\text {Cost }}$ was introduced to balance the need to reduce neutropenia with the desire to make the regimen easier to implement in terms of number of hospital visits and cost of the drug.

\section{Statistical methods}

Correlations between the predictions and the observed nadir timing and neutrophil counts, and their $95 \%$ confidence interval (CI), were calculated by Bland and Altman correction for dependence test (Bland and Altman, 1995). For these correlations, we allowed a time window of $\pm 6 \mathrm{~h}$, and calculated the mean neutrophil count for each data point. Note that clinical blood counts were monitored only once every few days. The positive predictive value was calculated by clinical confirmation of individual model's predictions of grade 3 or 4 neutropenia. Negative predictive values were similarly calculated for grade $0-2$ neutropenia. The significance of the positive and negative predictive values was calculated by Cohen's Kappa measure for agreement (Cohen, 1960).

\section{RESULTS}

\section{Model accuracy: comparison of clinical observations and model predictions}

The accuracy of the model was assessed in terms of three criteria: nadir timing (criterion i), neutrophil counts (criterion ii) and grade of toxicity per cycle (criterion iii). The predictive accuracy of 
the population-specific model, which was based on the clinical records of 12 patients from the training set, was assessed on a new set of $26 \mathrm{MBC}$ patients from the same populations (validation set), 16 receiving the tri-weekly regimen and 10 receiving the weekly regimen. The predictive accuracy of nadir timing in each treatment cycle was very high, with $r=0.99,95 \%$ CI: $0.98-1$ (criterion i; Figure 2E). A good overall prediction of individual neutrophil profiles (excluding the baseline measurements, which served as input) was found $r=0.62,95 \%$ CI: $0.58-0.69$ (criterion ii; Supplementary Figure S1), with a mean absolute error in neutrophil counts of \pm 383 neutrophils per $\mu$ l over all data points. The predictions for neutropenia grade of each patient (criterion iii) from the validation set were significant with positive and negative predictive values of grade 3-4 neutropenia, $86 \%$ and $83 \%$, respectively, $(\kappa=0.69, P<0.001)$. Specifically, the model predicted grade 3 or 4 neutropenia for 12 out of 14 patients and grade $0-2$ for 10 out of 12 patients who experienced grades $3-4$ or $0-2$ neutropenia, respectively. Simulation results of neutrophil profiles of four randomly chosen patients from the validation set as compared with their corresponding clinically observed neutrophil profiles are shown in Figure 2A-D.

Our model's predictions were also compared with an independent data set derived from clinical results of a phase I and PK clinical trial, in which the change of the neutrophil counts from the baseline of cancer patients, receiving Doc 5-115 $\mathrm{mg} \mathrm{m}^{-2}$ bi-weekly or tri-weekly, was recorded. The model's predictions were plotted as a function of the estimated area under the curve (AUC) of Doc plasma concentration and showed very good agreement with the clinical observations (Supplementary Figure S1).

\section{Validation of the model by literature data}

To further validate the population-specific model, its predictions for the approved $100 \mathrm{mg} \mathrm{m}^{-2}$ tri-weekly and $33 \mathrm{mg} \mathrm{m}^{-2}$ weekly Doc regimens for MBC (Rivera et al, 2008) were compared with clinical results from literature. For the $100 \mathrm{mg} \mathrm{m}^{-2}$ tri-weekly regimen, simulations predicted a nadir at day 7 post-Doc and recovery to baseline at ca. day 20 post-Doc, in patients whose baseline count was ca. 4250 neutrophils per $\mu$ l (Figure $3 \mathrm{~A}$, dasheddotted line). These results are supported by our clinical findings (Figure 2B-D) and by other Doc clinical-trial results in which nadir and recovery to baseline were recorded on days 6-10 and 16-22, respectively (Friberg et al, 2002; Ozawa et al, 2007). For the weekly $33 \mathrm{mg} \mathrm{m}^{-2}$ Doc schedule, the population-specific model predicts nadir coincidence with the subsequent Doc administration, although no higher toxicity than grade 0 or 1 neutropenia is predicted (neutrophil levels reach to ca. 1600 neutrophils per $\mu \mathrm{l}$ ). These results, implying that fractionation of the total Doc dose may relieve Doc-affected neutropenia, are corroborated by clinical observations (Gridelli et al, 2004; Gervais et al, 2005;
A
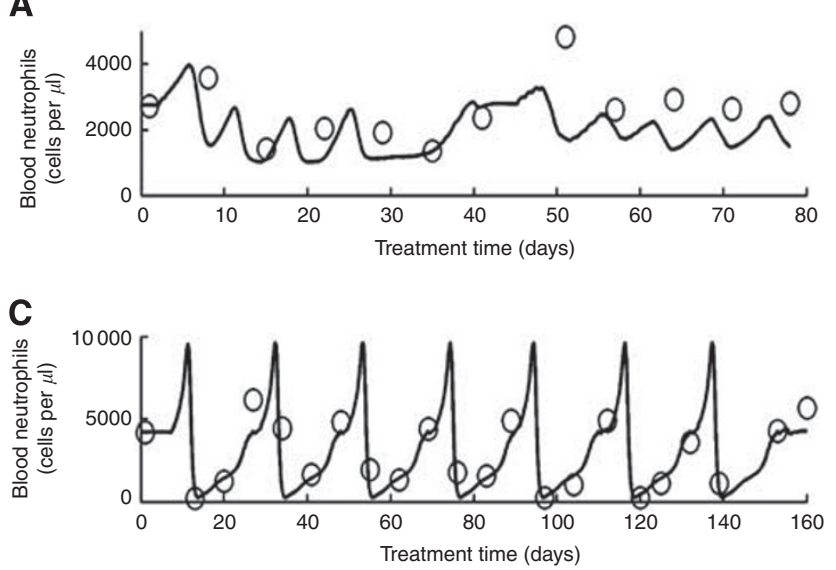

B
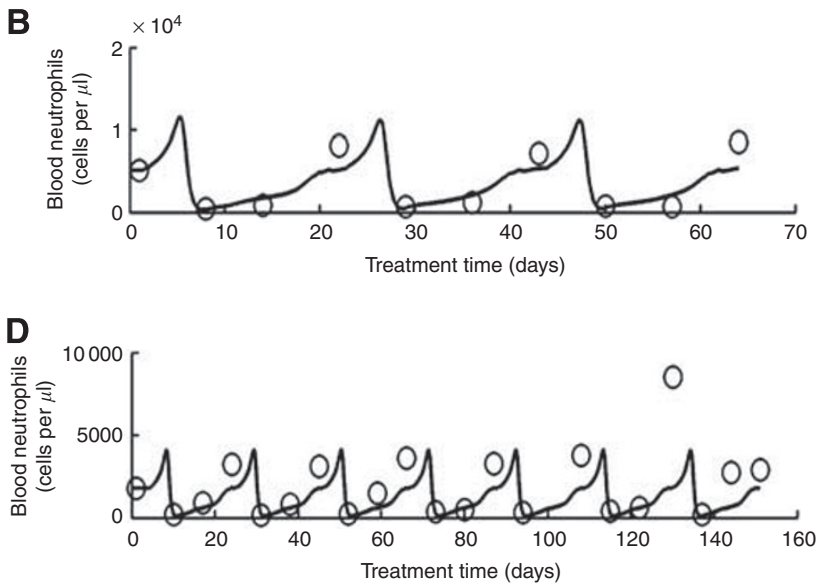

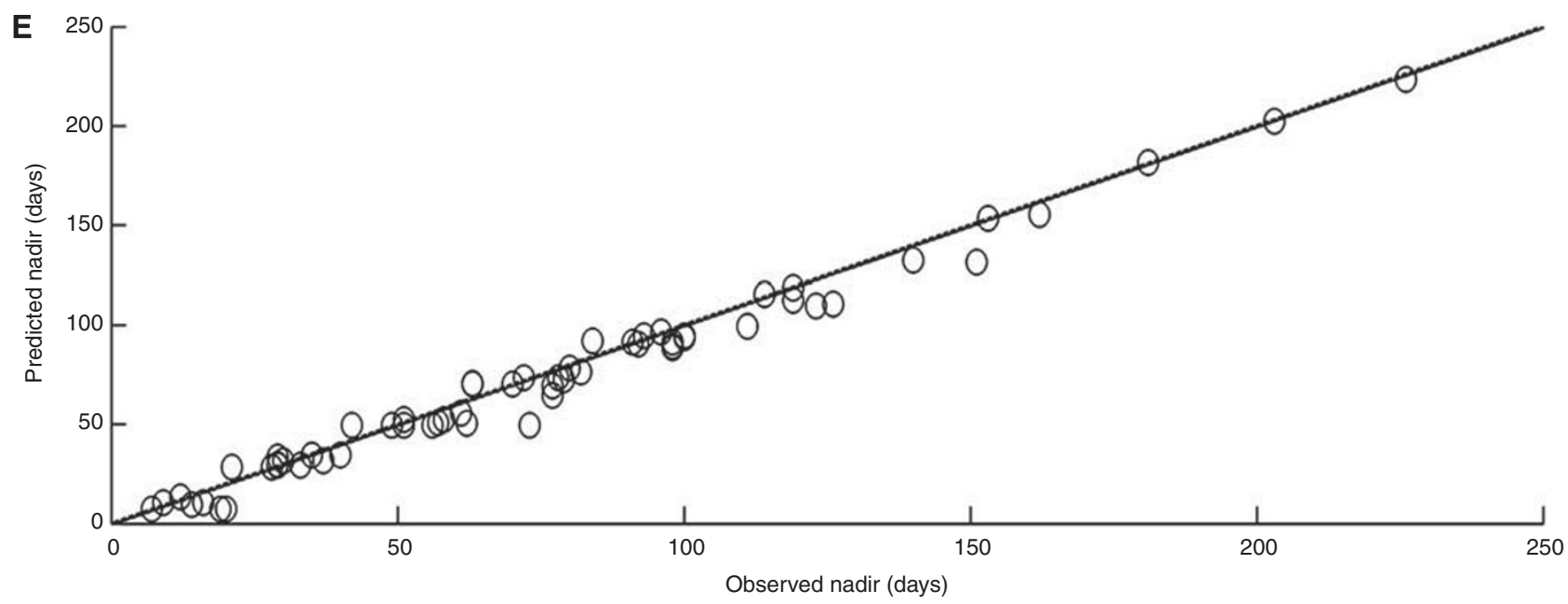

Figure 2 The model's predictions compared with clinical outcomes. An example of model-predicted neutrophil counts over time (solid lines) compared with the observed neutrophil counts (empty circles) of four MBC patients treated with different docetaxel schedules: $(\mathbf{A}) 25-35$ mg m ${ }^{-2}$ once weekly; (B-D) 100-75 $\mathrm{mg} \mathrm{m}^{-2}$ tri-weekly; (E) nadir days at each cycle, of patients receiving tri-weekly docetaxel (circles), model's predictions (abscissa), clinical observations (ordinate) and identity line (dashed line; here, the dashed line cannot be seen as it converges with the correlation line); $N=66$. Calculated correlation coefficient, $r=0.99$. 
A

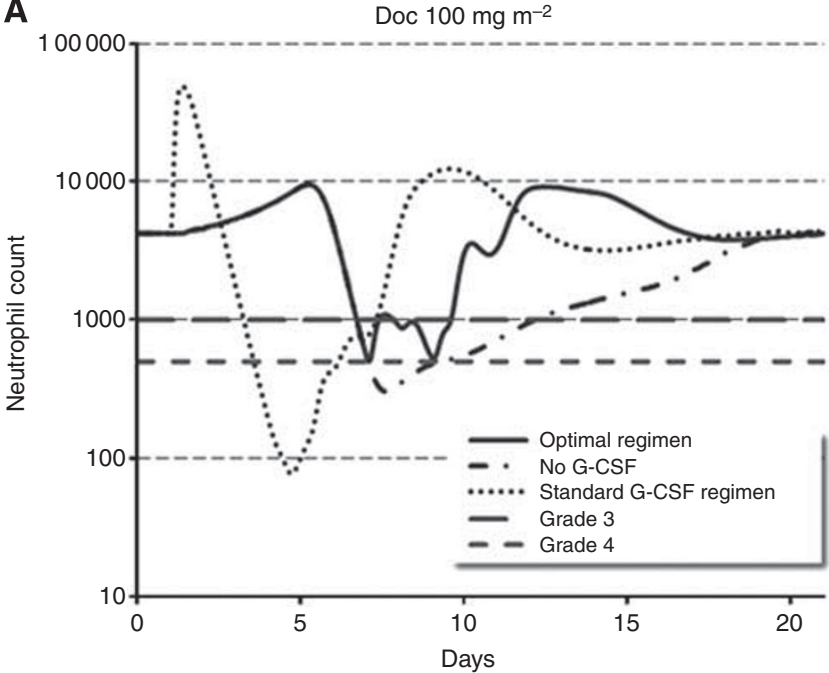

B

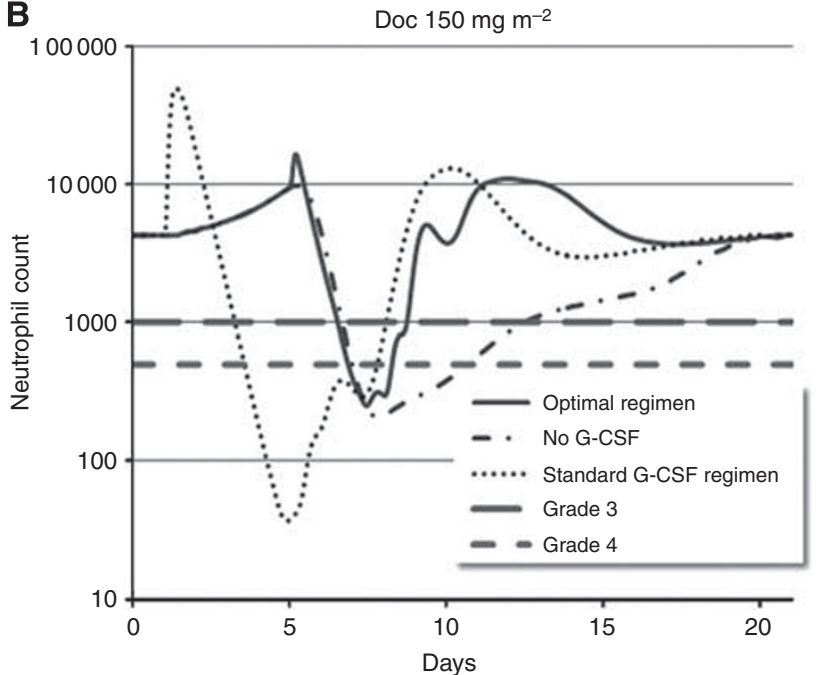

Figure 3 Neutrophils as a function of the docetaxel/G-CSF regimen. Treatment of MBC patients by G-CSF following a single administration of docetaxel was simulated using the population-adjusted physiological model. (A) Simulation results of blood neutrophil counts over time, following standard docetaxel administration of $100 \mathrm{mg} \mathrm{m}^{-2}$ tri-weekly; docetaxel only - no G-CSF (dashed-dotted line); standard G-CSF regimen: $300 \mu \mathrm{g}$ per day, QD × 5, starting I day after docetaxel (dotted line) or improved G-CSF regimen: $150 \mu \mathrm{g}$ per day, QD $\times 3$, starting on day 7 (solid line); upper limits of grades 3 and 4 neutropenia appear as black horizontal long and short dashed lines, respectively. (B) Simulation results of blood neutrophil counts over time, following dose intense (not approved) docetaxel administration of $150 \mathrm{mg} \mathrm{m}^{-2}$ tri-weekly; docetaxel only - no G-CSF (dashed-dotted line); standard G-CSF regimen $300 \mu \mathrm{g}$ per day, QD $\times 5$, starting I day after docetaxel (dotted line) or improved G-CSF regimen: $300 \mu \mathrm{g}$ per day, QD $\times 4$, starting on day 5 (solid line); upper limits of grades 3 and 4 neutropenia appear as described in $\mathbf{A}$.

Schuette et al, 2005; Rivera et al, 2008; Palmeri et al, 2008) and further support previous predictions concerning other chemotherapeutics (Agur et al, 2006).

\section{Effect of CYP3A-induced PK variability on model predictions}

It is well known that CYP3A affects the clearance of Doc (Hirth et al, 2000). We checked how the observed variability in this enzyme (Hirth et al, 2000) would influence the model's predictions concerning the extent of neutropenia in the individual patient. This was investigated by replacing the average patient clearance in the individual patient models with a randomly chosen value from the clinically observed range of clearance values (Hirth et al, 2000) and simulating neutropenic response. The differences were found to be insignificant in terms of nadir timing and duration of grade 3 or 4 neutropenia (see Supplementary Material for detailed explanation of methodology and Supplementary Table S2 for results).

\section{Optimising G-CSF treatment}

The validated model was integrated with the developed optimisation procedure, for identifying an improved schedule of G-CSF supportive care. This was done for both the standard tri-weekly Doc regimen (100 $\mathrm{mg} \mathrm{m}^{-2}$ tri-weekly) and for a higher Doc dosing regimen, which may increase treatment efficacy $\left(150 \mathrm{mg} \mathrm{m}^{-2}\right.$ tri-weekly). To this end, schedule optimisation was performed for a large number of patient models that vary in initial neutrophil counts and a large space of possible G-CSF schedules for each one of the two Doc treatments.

The goals of the optimisation was to identify a G-CSF schedule with minimal duration of neutropenia (grade 3), neutropenia level at the onset of the subsequent Doc administration higher than the grade 2 cutoff, and to minimise the amount of G-CSF and the number of G-CSF administrations.

The optimal supportive treatment found in the present study for average patients, having an initial count of 4250 neutrophils per $\mu \mathrm{l}$, given the standard tri-weekly Doc $100 \mathrm{mg} \mathrm{m}^{-2}$ consists of $300 \mu \mathrm{g}$
G-CSF applied on day 7 for 3 consecutive days (Figures 3A and $4 \mathrm{~A}$ ). This regimen causes a gradual and moderate decrease in neutrophil counts to grade 3 neutropenia lasting ca. 1 day. In contrast, the model predicts that the same Doc regimen supported by the standard G-CSF regimen of $300 \mu \mathrm{g}$ administered 1 day postDoc results in rapid mobilisation of the neutrophils from the BM post-mitotic reservoir into blood, followed by a radical blood neutrophil depletion (grade 4 neutropenia) occurring ca. day 3.5 and lasting 2.5 days (Figure $3 \mathrm{~A}$ ). The neutropenic response under the standard regimen is expected to be harsher than that with no G-CSF at all (Figure 3A). The G-CSF regimen, which was found optimal for borderline patients with an initial neutrophil count of 2000 neutrophils per $\mu \mathrm{l}$ (grade 1 cutoff) causing less than 2 days neutropenia when subjected to $100 \mathrm{mg}$ Doc, was G-CSF $300 \mu \mathrm{g}$, Q1D $\times 4$, starting on day 5 (data not shown). The latter regimen is less economical and slightly less beneficial for the average patient (initially having 4250 neutrophils per $\mu \mathrm{l}$, Figure 4B).

Applying a higher Doc dose $\left(150 \mathrm{mg} \mathrm{m}^{-2}\right)$ to patients with average neutrophil count (4250 neutrophils per $\mu$ l) or lower would be optimally supported by a regimen of G-CSF $300 \mu \mathrm{g}, \mathrm{Q} 1 \mathrm{D} \times 4$, starting on day 5. This regimen, which is expected to increase chemotherapy efficacy, yields slightly lower neutrophil levels, reaching grade 3 and 4 neutropenia that last ca. 2 days (Figures 3B and 4D). The standard G-CSF regimen, when applied after tri-weekly Doc $150 \mathrm{mg} \mathrm{m}^{-2}$, is expected to yield severe grade 4 neutropenia, occurring on day 3 and lasting over 4 days (Figure 3B). Similarly to the lower Doc dose, the neutropenia response is expected to be harsher under standard G-CSF regimen than with no G-CSF at all (Figure 3B). The more economical supportive regimen, G-CSF $300 \mu \mathrm{g}, \mathrm{Q} 1 \mathrm{D} \times 3$, starting on day 7 is expected to exert longer neutropenia (Figure 4C).

\section{DISCUSSION}

The computational method developed in this work relies on the quantitative predictive accuracy of the combined PK/PD and granulopoiesis model (Vainstein et al, 2005). We validated the 
A G-CSF onset day 7, Doc $100 \mathrm{mg} \mathrm{m}^{-2}$

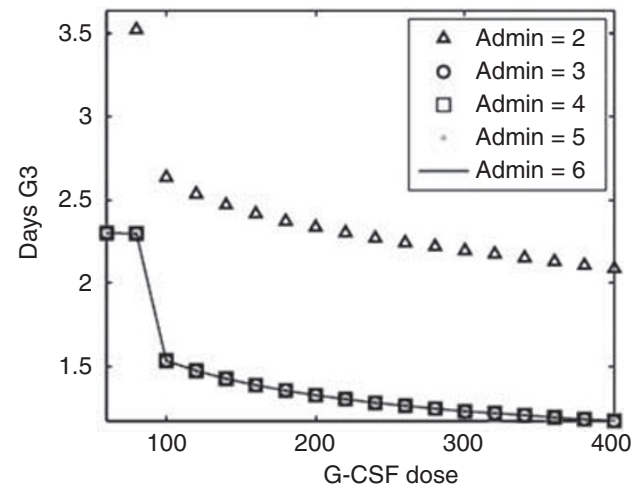

C G-CSF onset day 7, Doc 150 mg m²

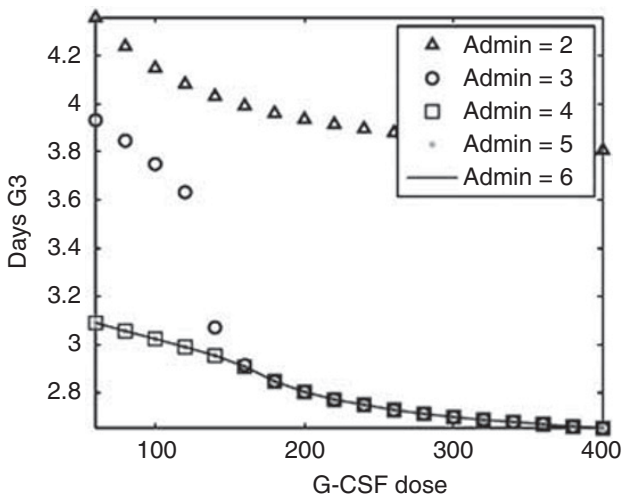

B

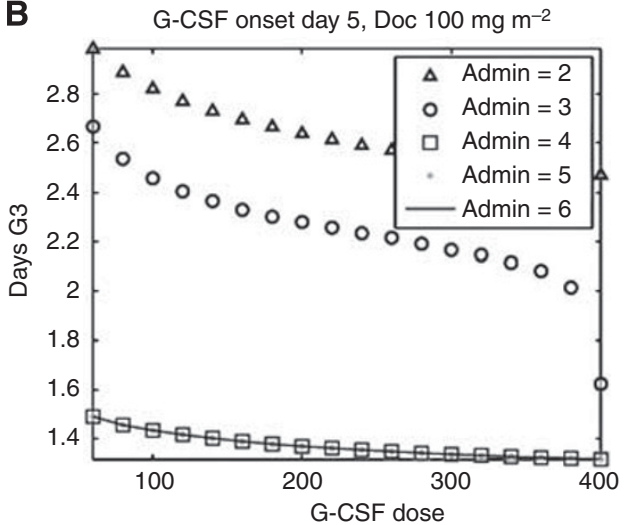

D G-CSF onset day 5, Doc $150 \mathrm{mg} \mathrm{m}^{-2}$

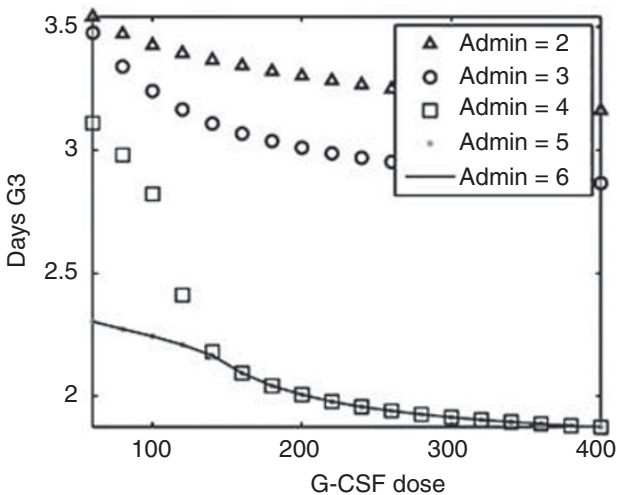

Figure 4 Optimisation of G-CSF regimen in an average patient (initial counts: 4250 neutrophils per $\mu$ ) treated by Doc; grade 3 neutropenia as a function

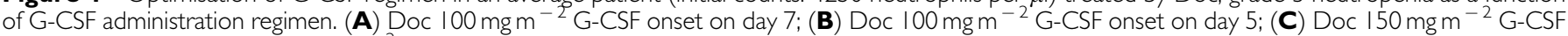
onset on day 7; (D) Doc $150 \mathrm{mg} \mathrm{m}^{-2}$ G-CSF onset on day 5. The number of daily G-CSF dosings appears in the window. The optimal G-CSG regimens, minimising both neutropenia and cost for Doc 100 and $150 \mathrm{mg} \mathrm{m}^{-2}$ are G-CSF $300 \mu \mathrm{g}$ per day, QID $\times 3$, on day 7 (A) and G-CSF $300 \mu \mathrm{g}$ per day, QID $\times 4$, on day $5(\mathbf{D})$, respectively.

combined model for chemotherapy-induced neutropenia in MBC patients. The ability to predict, before chemotherapy starts, the patient's timing of nadir and the grade of chemotherapyinduced neutropenia is of high clinical importance. Therefore, it is encouraging that the model proves accurate in predicting the nadir of the individual patient $(r=0.99)$, as well as grade 3 or 4 neutropenia in most patients experiencing it (positive predictive value of $86 \% ; \kappa=0.69$ ). Our combined model was validated for its high precision in predicting the observed nadir and all the recorded counts close to it. Model predictions of the true individual patient's nadir cannot be validated by the available clinical results, as clinical sampling was too sparse. That these predictions are probably reliable is suggested by the accuracy of our predictions in the neighbourhood of the nadir (Figure 2A-D). Moreover, our model is clinically useful, primarily because of its accurate predictions of the patient's grade and duration of toxicity; predicting the exact number of neutrophils at nadir is a less essential information. Nevertheless, we hope to conduct prospective trials to validate the accuracy of nadir prediction as well.

The validated model can be used for several means of clinical interest. First and foremost, based on limited clinical data, the model can predict neutrophil counts following drug administration. Using this feature in conjunction with our conceived optimisation method (see Patients and Methods section), personalised treatment protocols can be suggested, which are expected to minimise neutropenia at minimum cost, while maintaining or even increasing drug efficacy.
Simulating granulopoiesis with and without the standard G-CSF regimen, administered 1 day after chemotherapy application, suggests a decrease in the duration of grade 3 toxicity due to G-CSF administration. However, the standard G-CSF regimen may result in lower neutrophil counts with a nadir of less than 100 neutrophils per $\mu$ l as opposed to the nadir of 360 neutrophils per $\mu \mathrm{l}$ without G-CSF, and a prolonged duration of grade 4 toxicity (2.3 vs 1.4 days; Figure 3A).

This result can be interpreted by closely following the dynamics at the different granulopoiesis compartments, as simulated in our model. It is known that application of G-CSF has two major effects on granulopoiesis: (i) acceleration of neutrophil production and (ii) rapid release of neutrophils from BM reservoirs to blood, as opposed to the naturally occurring gradual neutrophil release (Vainstein et al, 2005). It is also known that Doc exerts its killing activity only on the early, replicating granulopoiesis compartments, as it is a cell-cycle-specific drug (Jordan and Wilson, 2004). Our model simulations show that administration of G-CSF immediately following Doc mobilises the BM neutrophil reservoirs into blood before and non-overlapping with the Doc-induced nadir. The now depleted BM reservoirs can no longer compensate for blood neutrophil shortage, and as a consequence, the nadir is more profound than that without G-CSF (Supplementary Figure S3).

These model predictions were corroborated by Meisenberg et al (1992) experimental study in non-human primates. The study showed that G-CSF treatment 1 day post-chemotherapy accelerates and aggravates the observed neutropenia. Applying G-CSF continuously, these authors observe a relatively fast recovery to baseline. 
Indeed, simulations of the treatment schedule (Meisenberg et al, 1992) showed grade 4 neutropenia with a fast recovery to baseline on day 10 (data not shown). The clinical implication of this finding should be further examined.

In the next stage of the study, we integrated the combined model with a new treatment optimisation procedure, to search for an optimal G-CSF regimen that would decrease both the extent and duration of neutropenia. For an average patient, having ca. 4250 neutrophils per $\mu \mathrm{l}$ and taking the standard Doc regimen, our optimisation procedure singled out an improved supportive regimen wherein G-CSF $300 \mu \mathrm{g}$ are administered on day 7 postDoc for 3 consecutive days. This regimen was found to release neutrophils from the post-mitotic BM reservoirs coinciding, and hence, moderating the potential neutrophil nadir caused by the Doc's damage to BM progenitors. Moreover, recovery to baseline was relatively rapid because of an efficient stimulatory effect of G-CSF on neutrophil production in the partially recovered BM. For a patient with borderline grade 1 neutropenia, the same supportive schedule was found to yield neutropenia that lasts ca. 3 days, rather than ca. 1 day for average patients. To obtain only 1 day neutropenia, borderline patients should receive more intensive G-CSF treatment, starting 5 days after chemotherapy.

To see if the higher median neutrophil level of the studied population may have affected the selected G-CSF schedule, we simulated patients whose baseline neutrophil counts ranged from 2000 to 8000 neutrophils per $\mu \mathrm{l}$ under three treatment alternatives: (i) no G-CSF, (ii) standard G-CSF regimen and (iii) our recommended G-CSF regimen. Results showed that under all three treatment options, toxicity linearly decreased with increasing baseline neutrophil counts. Over the whole range, the recommended G-CSF regimen yielded lower neutropenia duration than the two alternatives (data not shown).

Simulations of a virtual Doc-treated patient population that varied in the range $2000-8000$ neutrophils per $\mu$, or in the restricted range 2000-4250 neutrophils per $\mu \mathrm{l}$, theoretically confirmed the superiority of the G-CSF regimen suggested here. In these simulations, the average duration of grade 3 and 4 neutropenia was $2.76 \pm 0.44$ and $3.2 \pm 1.2$, respectively, under the recommended regimen as compared with $4.0 \pm 0.39$ and $4.4 \pm 0.8$, respectively, under the standard G-CSF regimen, and $5.2 \pm 2.17$ and $7.5 \pm 2.5$, respectively, under no support (data not shown). It should be borne in mind that these results are expected for Doc monotherapy only; combination therapies should be analysed separately. This holds true also for monotherapy by other chemotherapeutic agents; different drugs stimulate different hematopoietic changes and therefore are expected to be buffered by different G-CSF schedules. Indeed, our recommendations for optimal G-CSF scheduling following doxorubicin treatment were different than those following Doc (Vainstein et al, 2006).

The optimisation approach was further applied to the unapproved dose intense $150 \mathrm{mg} \mathrm{m}^{-2}$ tri-weekly Doc regimen to see if there was a way to give a more effective dose without compromising the safety of the patient. Our results show that neutropenia lasting less than 2 days was achieved by a supportive treatment regimen of $300 \mu \mathrm{g}$ of G-CSF administered on day 5 postDoc for 4 consecutive days. The same regimen applied to borderline patients resulted in a slightly longer neutropenia.

These results suggest a promising combination regimen for increased Doc dose intensity with optimally timed G-CSF support. The Doc dose intensity of the improved regimen is actually 1.5 -fold larger than the approved Doc intensity of $33 \mathrm{mg} \mathrm{m}^{-2}$ per week or $100 \mathrm{mg} \mathrm{m}^{-2}$ tri-weekly, and has been considered an overdose. However, the timing of G-CSF application with the suggested highintensity dosing or the approved $100 \mathrm{mg} \mathrm{m}^{-2}$ tri-weekly regimen is crucial; suboptimal timing is predicted to increase Doc-inflicted neutropenia. Moreover, our analysis suggests that the precise dosing regimen of G-CSF can be personalised to maximise its effect and minimise its cost.
Generally, our optimisation results suggest that the effect of G-CSF on neutropenia is determined by a combination of three factors: (i) the day of administration onset, (ii) the number of applications per cycle and (iii) the total daily dose. As is shown above, the largest reduction in neutropenia is achieved by three or four consecutive daily applications of no more than $300 \mu \mathrm{g} \mathrm{G}$-CSF. Higher doses of G-CSF and more than four daily applications are more costly and have negligible clinical benefit (Figure 4). These results are corroborated by our previous theoretical and experimental study, predicting and preclinically validating that four consecutive applications of thrombopoietin will optimally stimulate thrombopoiesis (Skomorovski et al, 2003).

In patients with reduced neutrophil counts or in average patients under severe chemotherapy, neutropenia is expected to be slightly longer (by 1 day or less) under G-CSF $300 \mu \mathrm{g}$ Q1D $\times 3$, starting on day 7 , than when four G-CSF dosings are applied, starting on day 5. However, the slightly prolonged neutropenic toxicity of the regimen started on day 7 is expected to be traded off by its reduced cost, and possibly also the side effects, as well as by the increased ease of its application. The choice of the best regimen for the patient is a prerogative of the doctor.

Our work points out the crucial effect of timing of G-CSF application with respect to chemotherapy. We suggest that when optimally applied (see above), G-CSF may significantly moderate the chemotherapy-caused neutrophil nadir. This result might have important implications for clinical oncology (Smith et al, 2006), enabling more efficacious support to highly cytotoxic drugs. In another work, optimal G-CSF application has been considered in patients suffering severe chemotherapy-induced neutropenia at nadir. However, that work ignored the effect of the timing of G-CSF application and the possibility that when optimally timed, G-CSF might alleviate the harsh trough in neutrophil count caused by chemotherapy (Shochat and Rom-Kedar, 2008).

Pegylated G-CSF will be analysed separately because of its distinct PK properties. It should be borne in mind that the main reason for administering pegylated G-CSF is its prolonged effect. If, indeed, this is the only significant PK/PD difference between the two forms of the drug, then based on our analysis, we expect that initiating pegylated G-CSF later than day 2 after chemotherapy would be preferable. Indeed, Zwick et al (2011) suggest that Peg-F should be given on day 4 instead of day 2 post-chemotherapy, as it results in less leukocytopenia, less infections and less therapyassociated deaths.

In our work, we have focused on two representative patients, one with normal and the other with low neutrophil counts, considered at the cutoff point of neutropenia. However, the methodology developed here is easily applicable to individual patient models in the case wherein individual data on initial neutrophil count and Doc clearance is available, ultimately leading to a personal predictive tool for tailoring personal treatment.

Our simulation results suggest that the introduction of CYP3Ainduced PK variability (Hirth et al, 2000) has little effect on the model's predictions concerning the timing of nadir and the duration of grade 3 or 4 neutropenia (see analysis in Supplementary Materials). On the basis of these results, one can conclude that the model's predictions under the assumption of population average Doc clearance are robust to $\mathrm{PK}$ inter-patient variability, which is due to the variability in CYP3A. Other covariates such as $\alpha-1$ acid glycoprotein data (Urien et al, 1996), age, gender and so on are known to affect the clearance of Doc (Bruno et al, 2003). However, simulations of the model over the corresponding wider range of clearance values (Bruno et al, 2003) reveal that like the $\mathrm{CYP} 3 \mathrm{~A}$ induced clearance variability, these other covariates, even though they lead to an overall variability in AUC, are not expected to have a large effect on nadir timing and length of grade 3 or 4 toxicity (data not shown).

Our model differs both from current PK/PD models and from phenomenological models, and even from other mechanistic 
models. For example, Engel et al (2004) provide a detailed mechanistic granulopoiesis model to account for the short- and long-term effects of combined chemotherapy and G-CSF. Our model represents a step forward in this type of granulopoiesis modelling, mainly as it accounts for cell cycle phase transition. This feature is a prerequisite for predicting long-term patient response to cell cycle phase-specific drugs, such as the individual hour of nadir in each treatment cycle, or the differential effects of these drugs as compared with the effects of cell cycle phase nonspecific drugs. To the best of our knowledge, our model is the first to be used for predicting the personal response to mono or combination therapies over extended treatment periods. Therefore, it can also predict the personal cumulative toxic effects of drugs on granulopoiesis. Other chemotherapeutic and chemosupportive agents can be adapted to our model, including pegylated G-CSF.

Determining the improved administration schedules of chemotherapy and supporting drugs is a tedious task, and the insufficiency of current predictive tools is a great impediment to treatment personalisation. For example, lack of methods for predicting the time of chemotherapy-induced nadir in individual patients under cytotoxic chemotherapy often impedes timely application of G-CSF. This and other treatment personalisation issues can be addressed by the use of our drug regimen optimisation methodology, according to which phenomenological

\section{REFERENCES}

Agur Z, Hassin R, Levy S (2006) Optimizing chemotherapy scheduling using local search heuristics. Operations Res 54: 829-846

Alenzi FQ, Alenazi BQ, Ahmad SY, Salem ML, Al-Jabri AA, Wyse RK (2009) The haemopoietic stem cell: between apoptosis and self renewal. Yale $J$ Biol Med 82(1): 7-18

Baker SD, Zhao M, Lee CK, Verweij J, Zabelina Y, Brahmer JR, Wolff AC, Sparreboom A, Carducci MA (2004) Comparative pharmacokinetics of weekly and every-three-weeks docetaxel. Clin Cancer Res 10(6): 1976-1983

Bland JM, Altman DG (1995) Calculating correlation coefficients with repeated observations: part 2-correlation between subjects. $B m j$ 310(6980): 633

Bonate P (2006) Pharmacokinetic-Pharmacodynamic Modeling and Simulation. Springer Science and Business Media Inc: New York

Bruno R, Hille D, Riva A, Vivier N, ten Bokkel Huinnink WW, van Oosterom AT, Kaye SB, Verweij J, Fossella FV, Valero V, Rigas JR, Seidman AD, Chevallier B, Fumoleau P, Burris HA, Ravdin PM, Sheiner LB (1998) Population pharmacokinetics/pharmacodynamics of docetaxel in phase II studies in patients with cancer. J Clin Oncol 16(1): 187-196

Bruno R, Olivares R, Berille J, Chaikin P, Vivier N, Hammershaimb L, Rhodes GR, Rigas JR (2003) Alpha-1-acid glycoprotein as an independent predictor for treatment effects and a prognostic factor of survival in patients with non-small cell lung cancer treated with docetaxel. Clin Cancer Res 9(3): 1077-1082

Bruno R, Vivier N, Vergniol JC, De Phillips SL, Montay G, Sheiner LB (1996) A population pharmacokinetic model for docetaxel (Taxotere): model building and validation. J Pharmacokinet Biopharm 24(2): 153-172

Cameron D (2009) Management of chemotherapy-associated febrile neutropenia. Br J Cancer 101(Suppl 1): S18-S22

Cohen J (1960) A coefficient of agreement for nominal scales. Educ Psychol Measurement 20(1): 37-46

Cortes JE, Pazdur R (1995) Docetaxel. J Clin Oncol 13(10): 2643-2655

Crawford J, Dale DC, Lyman GH (2004) Chemotherapy-induced neutropenia: risks, consequences, and new directions for its management. Cancer 100(2): 228-237

Dingli D, Antal T, Traulsen A, Pacheco JM (2009) Progenitor cell selfrenewal and cyclic neutropenia. Cell Prolif 42(3): 330-338

Engel C, Scholz M, Loeffler M (2004) A computational model of human granulopoiesis to simulate the hematotoxic effects of multicycle polychemotherapy. Blood 104(8): 2323-2331

Esbensen K (2002) Multivariate Data Analysis-In Practice. 5 edn. CAMO ASA: Norway

Foley C, Mackey MC (2009) Mathematical model for G-CSF administration after chemotherapy. J Theor Biol 257(1): 27-44 and mechanistic models are integrated under one framework and simulated to rank a regimen's efficacy and toxicity.

We hope that this work will be a step forward towards routine implementation of mathematical models for personalising oncology schedules, and for replacing trial and error methods in drug development by more rational methods leading to efficacious administration of drugs with increased patients' compliance.

\section{ACKNOWLEDGEMENTS}

This study was partly supported by the Office of the Chief Scientist of Israel's Ministry of Industry, Labour and Trade, and the Cancer Research UK pilot project Grant (C22276/A7233). We thank Dorit Dror for secretarial help.

\section{Author contributions}

$\mathrm{MK}, \mathrm{OV}, \mathrm{RB}$ and $\mathrm{ZA}$ designed the study; OV and MK constructed the PK/PD model; SA, WM, AM and SC collected clinical data; $\mathrm{OV}, \mathrm{OA}, \mathrm{OI}, \mathrm{VV}$ and $\mathrm{ZA}$ constructed the optimisation procedure; $\mathrm{OV}$ and OA simulated the models; OV, OA and ZA wrote the article.

Supplementary Information accompanies the paper on British Journal of Cancer website (http://www.nature.com/bjc)

Friberg LE, Henningsson A, Maas H, Nguyen L, Karlsson MO (2002) Model of chemotherapy-induced myelosuppression with parameter consistency across drugs. J Clin Oncol 20(24): 4713-4721

Gervais R, Ducolone A, Breton JL, Braun D, Lebeau B, Vaylet F, Debieuvre D, Pujol JL, Tredaniel J, Clouet P, Quoix E (2005) Phase II randomised trial comparing docetaxel given every 3 weeks with weekly schedule as second-line therapy in patients with advanced non-small-cell lung cancer (NSCLC). Ann Oncol 16(1): 90-96

Gridelli C, Frontini L, Barletta E, Rossi A, Barzelloni ML, Scognamiglio F, Guida C, Gatani T, Fiore F, De Bellis M, Marfella A, Manzione L (2000) Single agent docetaxel plus granulocyte-colony stimulating factor (GCSF) in previously treated patients with advanced non small cell lung cancer. A phase II study and review of the literature. Anticancer Res 20(2B): 1077-1084

Gridelli C, Gallo C, Di Maio M, Barletta E, Illiano A, Maione P, Salvagni S, Piantedosi FV, Palazzolo G, Caffo O, Ceribelli A, Falcone A, Mazzanti P, Brancaccio L, Capuano MA, Isa L, Barbera S, Perrone F (2004) A randomised clinical trial of two docetaxel regimens (weekly vs 3 week) in the second-line treatment of non-small-cell lung cancer. The DISTAL 01 study. Br J Cancer 91(12): 1996-2004

Hardy CL, Balducci L (1985) Hemopoietic alterations of cancer. Am J Med Sci 290(5): 196-205

Hirth J, Watkins PB, Strawderman M, Schott A, Bruno R, Baker LH (2000) The effect of an individual's cytochrome CYP3A4 activity on docetaxel clearance. Clin Cancer Res 6(4): 1255-1258

Jerri AJ (1979) Correction to "The Shannon sampling theorem\&\#8212;Its various extensions and applications: A tutorial review". Proc IEEE 67(4): 695-695

Jordan MA, Wilson L (2004) Microtubules as a target for anticancer drugs. Nat Rev Cancer 4(4): 253-265

Krell D, Jones AL (2009) Impact of effective prevention and management of febrile neutropenia. Br J Cancer 101(Suppl 1): S23-S26

Kroese D, Porotsky S, Rubinstein R (2006) The cross-entropy method for continuous multi-extremal optimization. Method Comput Applied Probab 8: 383-407

Mackey MC, Aprikyan AA, Dale DC (2003) The rate of apoptosis in post mitotic neutrophil precursors of normal and neutropenic humans. Cell Prolif 36(1): 27-34

Meisenberg BR, Davis TA, Melaragno AJ, Stead R, Monroy RL (1992) A comparison of therapeutic schedules for administering granulocyte colony-stimulating factor to nonhuman primates after high-dose chemotherapy. Blood 79(9): 2267-2272

Montero A, Fossella F, Hortobagyi G, Valero V (2005) Docetaxel for treatment of solid tumours: a systematic review of clinical data. Lancet Oncol 6(4): 229-239 
Ostby I, Rusten LS, Kvalheim G, Grottum P (2003) A mathematical model for reconstitution of granulopoiesis after high dose chemotherapy with autologous stem cell transplantation. J Math Biol 47(2): 101-136

Ozawa K, Minami H, Sato H (2007) Population pharmacokinetic and pharmacodynamic analysis for time courses of docetaxel-induced neutropenia in Japanese cancer patients. Cancer Sci 98(12): 1985-1992

Pacheco JM, Traulsen A, Dingli D (2009) The allometry of chronic myeloid leukemia. J Theor Biol 259(3): 635-640

Paciucci PA, Raptis G, Bleiweiss I, Weltz C, Lehrer D, Gurry R (2002) Neo-adjuvant therapy with dose-dense docetaxel plus short-term filgrastim rescue for locally advanced breast cancer. Anticancer Drugs 13(8): 791-795

Palmeri L, Vaglica M, Palmeri S (2008) Weekly docetaxel in the treatment of metastatic breast cancer. Ther Clin Risk Manag 4(5): 1047-1059

Rivera E, Mejia JA, Arun BK, Adinin RB, Walters RS, Brewster A, Broglio KR, Yin G, Esmaeli B, Hortobagyi GN, Valero V (2008) Phase 3 study comparing the use of docetaxel on an every-3-week versus weekly schedule in the treatment of metastatic breast cancer. Cancer 112(7): 1455-1461

Schmitz S, Franke H, Brusis J, Wichmann HE (1993) Quantification of the cell kinetic effects of G-CSF using a model of human granulopoiesis. Exp Hematol 21(6): 755-760

Schmitz S, Loeffler M, Jones JB, Lange RD, Wichmann HE (1990) Synchrony of bone marrow proliferation and maturation as the origin of cyclic haemopoiesis. Cell Tissue Kinet 23(5): 425-442

Schuette W, Nagel S, Blankenburg T, Lautenschlaeger C, Hans K, Schmidt EW, Dittrich I, Schweisfurth H, von Weikersthal LF, Raghavachar A, Reissig A, Serke M (2005) Phase III study of second-line chemotherapy for advanced non-small-cell lung cancer with weekly compared with 3-weekly docetaxel. J Clin Oncol 23(33): 8389-8395

Shannon CE (1949) Communication in the presence of noise. Proc IRE 37(1): $10-21$

Shochat E, Rom-Kedar V (2008) Novel strategies for granulocyte colonystimulating factor treatment of severe prolonged neutropenia suggested by mathematical modeling. Clin Cancer Res 14(20): 6354-6363
Shochat E, Stemmer SM, Segel L (2002) Human haematopoiesis in steady state and following intense perturbations. Bull Math Biol 64(5): 861-886

Skomorovski K, Harpak H, Ianovski A, Vardi M, Visser TP, Hartong SC, van Vliet HH, Wagemaker G, Agur Z (2003) New TPO treatment schedules of increased safety and efficacy: pre-clinical validation of a thrombopoiesis simulation model. Br J Haematol 123(4): 683-691

Smith TJ, Khatcheressian J, Lyman GH, Ozer H, Armitage JO, Balducci L, Bennett CL, Cantor SB, Crawford J, Cross SJ, Demetri G, Desch CE, Pizzo PA, Schiffer CA, Schwartzberg L, Somerfield MR, Somlo G, Wade JC, Wade JL, Winn RJ, Wozniak AJ, Wolff AC (2006) 2006 update of recommendations for the use of white blood cell growth factors: an evidence-based clinical practice guideline. J Clin Oncol 24(19): 3187-3205

Urien S, Barre J, Morin C, Paccaly A, Montay G, Tillement JP (1996) Docetaxel serum protein binding with high affinity to alpha 1-acid glycoprotein. Invest New Drugs 14(2): 147-151

Vainstein V, Ginosar Y, Shoham M, Ranmar DO, Ianovski A, Agur Z (2005) The complex effect of granulocyte colony-stimulating factor on human granulopoiesis analyzed by a new physiologically-based mathematical model. J Theor Biol 234(3): 311-327

Vainstein V, Ginosaur Y, Shoham M, Ianovski A, Rabinovich A, Kogan Y, Selitser Z, Agur Z (2006) Improving cancer therapy by doxorubicin and granulocyte colony-stimulating factor: insights from a computerized model of human granulopoiesis. Math Model Nat Phenom 1(2): 70-80

van Zuylen L, Sparreboom A, van der Gaast A, van der Burg ME, van Beurden V, Bol CJ, Woestenborghs R, Palmer PA, Verweij J (2000) The orally administered P-glycoprotein inhibitor R101933 does not alter the plasma pharmacokinetics of docetaxel. Clin Cancer Res 6(4): 1365-1371

Zwick C, Hartmann F, Zeynalova S, Poschel V, Nickenig C, Reiser M, Lengfelder E, Peter N, Schlimok G, Schubert J, Schmitz N, Loeffler M, Pfreundschuh M (2011) Randomized comparison of pegfilgrastim day 4 versus day 2 for the prevention of chemotherapy-induced leukocytopenia. Ann Oncol 22(8): 1872-1877

This work is published under the standard license to publish agreement. After 12 months the work will become freely available and the license terms will switch to a Creative Commons Attribution-NonCommercial-Share Alike 3.0 Unported License. 